\title{
Effect of low gravitational stimulation on the perception of target elevation: Role of spatial expertise
}

\author{
JÉRÔME CARRIOT and CORINNE CHARLES \\ Centre de Recherches du Service de Santé des Armées, Grenoble, France \\ and Université Joseph Fourier, Grenoble, France \\ ANNE-FABIENNE HUFFENUS and VINCENT NOUGIER \\ Université Joseph Fourier, Grenoble, France \\ and \\ CHRISTIAN RAPHEL and CORINNE CIAN \\ Centre de Recherches du Service de Santé des Armées, Grenoble, France
}

\begin{abstract}
To examine the interindividual differences in the judgment of the visually perceived eye level (VPELupright position) and of the visually perceived apparent zenith (VPAZ-supine position) when the subject is subjected to low gravitational-inertial force (GIF), we independently altered GIF in two different populations: control subjects and spatial experts. Subjects were instructed to set a luminous target to the eye level while they were in total darkness and motionless or undergoing low radial acceleration with respect to the threshold of the otolithic system $\left(0.015-1.67 \mathrm{~m} / \mathrm{sec}^{2}\right.$ for the VPEL and $0.55-2.19 \mathrm{~m} / \mathrm{sec}^{2}$ for the VPAZ, respectively). Results showed that (1) low GIFs, close to those met during daily life, induced an eye level lowering in the upright and supine positions for the control group, and (2) the spatial expertise modified the influence of low GIF. Whereas an oculogravic illusion was found for the control group, this phenomenon was absent (VPAZ) or weaker (VPEL) for the spatial experts. Thus, the relations that the subjects maintain with their spatial environment and the knowledge acquired through experience modify the processing of sensory information and the perceptive construction resulting from it. The interindividual differences in sensitivity to the oculogravic illusion are discussed in terms of sensory dominance and of a better efficiency in the use of the available sensory information.
\end{abstract}

Most people can determine whether a given target is above or below the level of their eyes. In the dark, they can easily position the target so that it appears to be at eye level. For a usual direction of the head axis (i.e., vertical), a target is considered to be at eye level when an imaginary line connecting the target to the eyes is perpendicular to the direction of gravity. The angular deviation between the visual target set to appear at eye level and this horizontal plane defines the visually perceived eye level (VPEL; Dizio, Li, Lackner, \& Matin, 1997; Li \& Matin, 1993, 1995; Matin \& Li, 1992, 1994, 1995).

The VPEL is strongly influenced by the variation of the gravitational-inertial forces (GIFs) acting on the subject. This is the case when an upright subject faces toward the

The authors are grateful to the Instrumentation and Technological Research Service of the Centre de Recherches du Service de Santé des Armées for the construction of the device used in this experiment and for their technical expertise and assistance. They are also grateful to anonymous reviewers for their helpful comments and suggestions. Correspondence may be directed to C. Cian, CRSSA, Département des facteurs humains, BP 87, 38702 La Tronche Cedex, France (e-mail: corinnecian@ crssa.net). center of a centrifuge that rotates at a steady velocity for some time. A target that remains at true eye level appears to be above its true location when the observer is exposed to a change in both magnitude and direction of GIFs. This so-called oculogravic illusion induces a lowering of the VPEL (Cohen, 1973; Cohen, Stoper, Welch, \& DeRoshia, 2001; Graybiel, 1952; Schöne, 1964; Whiteside, Graybiel, $\&$ Niven, 1965). This phenomenon has also been shown for very low variations of GIFs $\left(9.81001 \mathrm{~m} / \mathrm{sec}^{2}\right)$ produced by radial accelerations - that is, from 0.01 to $1.67 \mathrm{~m} / \mathrm{sec}^{2}$ (Raphel \& Barraud, 1994; Raphel, Barraud, Koessler, \& Cian, 1996). However, for the weaker variations of GIFs (i.e., GIF $\leq 1.05 \mathrm{G}$ ), the relationship between the radial acceleration and the lowering of VPEL was logarithmic (Raphel \& Barraud, 1994; Raphel et al., 1996) whereas for higher changes of GIFs (i.e., GIF $\geq 1.25 \mathrm{G}$ ), this relationship was linear (Cohen, 1973; Correia, Hixon, \& Niven, 1968). The differences between the linear function and the logarithmic function may be caused by differences in sense organ stimulation (Raphel \& Barraud, 1994). Indeed, higher GIF changes produced by acceleration result in an altered stimulation of the otolith organs of the vestibular system, as well as of the muscle and cutaneous 
proprioceptors (Cohen, 1981; Miller \& Graybiel, 1966; Schöne, 1964; Wade \& Schöne, 1971), whereas bodily senses affected by lowest GIFs are restricted. Indeed, the very limited variations of GIFs exclude any tactile and kinesthetic origin because the variations in subject's weight are negligible with respect to the sensitivity threshold of the tactile and kinesthetic organs. Thus, the lowering of the VPEL is probably due to the stimulation of the otolithic system alone.

In the range of very low gravitational-inertial stimulation, errors were also reported in the perceived elevation of a visual target that is viewed by an observer in a supine position (Raphel, Cian, Barraud, \& Micheyl, 2001). When the body and head axes are perpendicular to the direction of gravity (i.e., in the supine position), the subjective perception of the eye level corresponds to the plane as being parallel to the direction of gravity (visually perceived apparent zenith:VPAZ). Changes of the gravitational-inertial field induce a VPAZ lowering similar to that observed for the VPEL - that is, a shift in the direction of the resultant GIF (Figure 1). Nevertheless, the theoretical threshold of sensitivity to radial accelerations is higher in the supine $\left(0.38 \mathrm{~m} / \mathrm{sec}^{2}\right)$ than in the upright position $\left(0.0006 \mathrm{~m} / \mathrm{sec}^{2}\right)$. This difference may be related to a difference in sense organ stimulation. Considering the spatial position of the otolithic system - that is, the orientation of the utricular membrane close to the horizontal plane when the head is held erect whereas the saccular maculae is oriented globally nearly vertically (Citek \& Ebenholtz, 1996; Curthoys et al., 1999; Kelly, 1991) — and the shearing force acting on it, it has been assumed that the utricular membrane is involved in the eye level lowering in the upright position (Raphel \& Barraud, 1994) and in the saccular maculae in the supine position (Raphel et al., 2001). Within an ecological framework of sensory signals processing, the relevance and the processing of the utricular and saccular signals may depend on the conditions under which these sensory organs are usually stimulated. When subjects are standing upright, the utricular membrane is primarily activated by backward and forward movements of the head under accelerations ranging from zero to several meters per seconds squared. Under such conditions, the detection threshold of linear accelerations should be very low. The saccular membrane is continuously submitted to gravity (i.e., $9.81 \mathrm{~m} / \mathrm{sec}^{2}$ ), and variations in the gravitational direction are usually very small. Under such conditions, the detection threshold to accelerations should be higher.

According to this framework, the functional capacities of the system are also determined by the knowledge acquired through experience, suggesting interindividual differences in the perceptivospatial ability. In the same way, Matin $(1982,1986)$ and Matin and Li (1992) underlined this interindividual variability in their model of VPEL evaluation (which is similar to that proposed by Stoper \& Cohen, 1989, 1991). When an observer views a single target in the dark, this model can be summarized as follows: VPEL $=B_{\mathrm{s}}+B_{\mathrm{o}}$ (Matin \& Fox, 1989), where $B_{\mathrm{s}}$ expresses the stimulus inputs and $B_{\mathrm{o}}$ expresses the relative weight each individual attributes to the propriosomaesthetic information. This differential individual weight could find an explanation in the sensibility with which the subjects perceive a variation of sensory state. This sensibility would depend on the comparison of the signals related to body orientation with predictive internal models that specify expected sensory configurations (Berthoz \& Viaud-Delmon, 1999). From a body perception and control orientation point of view, these internal representations are elaborated on the basis of subjects' experience (Lackner, 1992; Lestienne \& Gurfinkel, 1988; Young, Mendoza, Groleau, \& Wojcik, 1996). The individual dif-

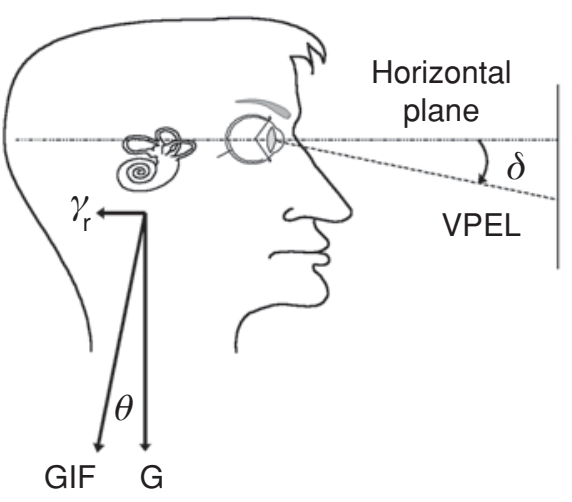

(Raphel \& Barraud, 1994)

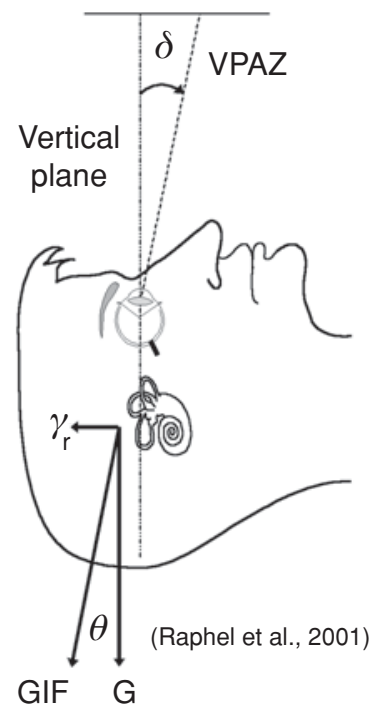

Figure 1. Position of the vestibular system with respect to the conditions of centrifugation in the upright (left panel) and supine (right panel) positions. 
ferences of apparent target elevation would be partially dependent on the subjects' experience of GIF variations.

The principal aim of this study was to understand how the relation that the subject maintains with the spatial environment - that is, the knowledge acquired through accumulated experience-modifies the processing of sensory information and the perceptive construction resulting from it. To achieve this goal, we replicated the experiments of Raphel and Barraud (1994) and Raphel et al. (2001) - that is, their studies of low gravitational-inertial stimulation applied to subjects in the upright and in the supine positions, respectively-with two different populations: (1) subjects without sports expertise and (2) highlevel acrobats (trampolinists). More precisely, we investigated to which extent the spatial expertise modifies the influence of low gravitational-inertial disturbances on the eye level. Indeed, a possible interpretation of a good spatial control consists in attributing to the acrobats' perception a predictive character (Berthoz, 1997). Acrobats, as probably any experts in motor skills requiring a fine control of body orientation, are trained to face high postural constraints in some particular environments in which the sensory redundancy is often limited (e.g., disrupted somatosensory or visual inputs). Unconsciously, they would have improved the functional characteristics of the different sensory systems and/or would have learned perceptual strategies that consist of picking up and associating the relevant information still available (Bringoux, Marin, Nougier, Barraud, \& Raphel, 2000). It was hypothesized that the gravitational-inertial disturbances do not induce the same negative consequences on the VPEL and VPAZ for spatial experts and for control subjects. Under low gravitational-inertial stimulation, the radial acceleration would induce no VPEL and VPAZ shift for the experts, or if this shift was present, it would be smaller than for the control subjects.

\section{METHOD}

\section{Subjects}

Forty male subjects participated in this study. They had normal vision and were free of any apparent vestibular disorders. All of them were naive about the purpose of the experiment and gave informed consent in compliance with the Huriet Law (i.e., Helsinki Convention), which governs and regulates human experimentation in France. Subjects were differently skilled in trampoline: 20 subjects recruited in a training camp (ages 18 to 26) practiced trampoline at a national or international level, which means more than 10 years of intensive practice, generally initiating with gymnastics and then moving to trampoline (expert group) and 20 subjects (ages 20 to 36) had no special sports expertise (control group). Twentyfour subjects participated in the VPEL experiment (12 expert and 12 control subjects). The last 16 subjects participated in the VPAZ experiment ( 8 expert and 8 control subjects). None of these subjects were familiar with the experiment. Furthermore, expert gymnasts are habituated to variations in gravitational force; that is, they are submitted, in their daily sports, to strong and sudden changes of the angular and linear accelerations, but should not be more familiar with control subjects to linear constant velocity stimulation.

\section{Apparatus}

The basic apparatus was a centrifuge that could rotate at a constant velocity between 0 and $150^{\circ} / \mathrm{sec}$. In the VPEL experiment, subjects were seated upright facing the yaw axis of rotation (Figure 2, left panel). In the VPAZ experiment, subjects were lying on a horizontal plane that rotated around the chest to spine (Gx) axis

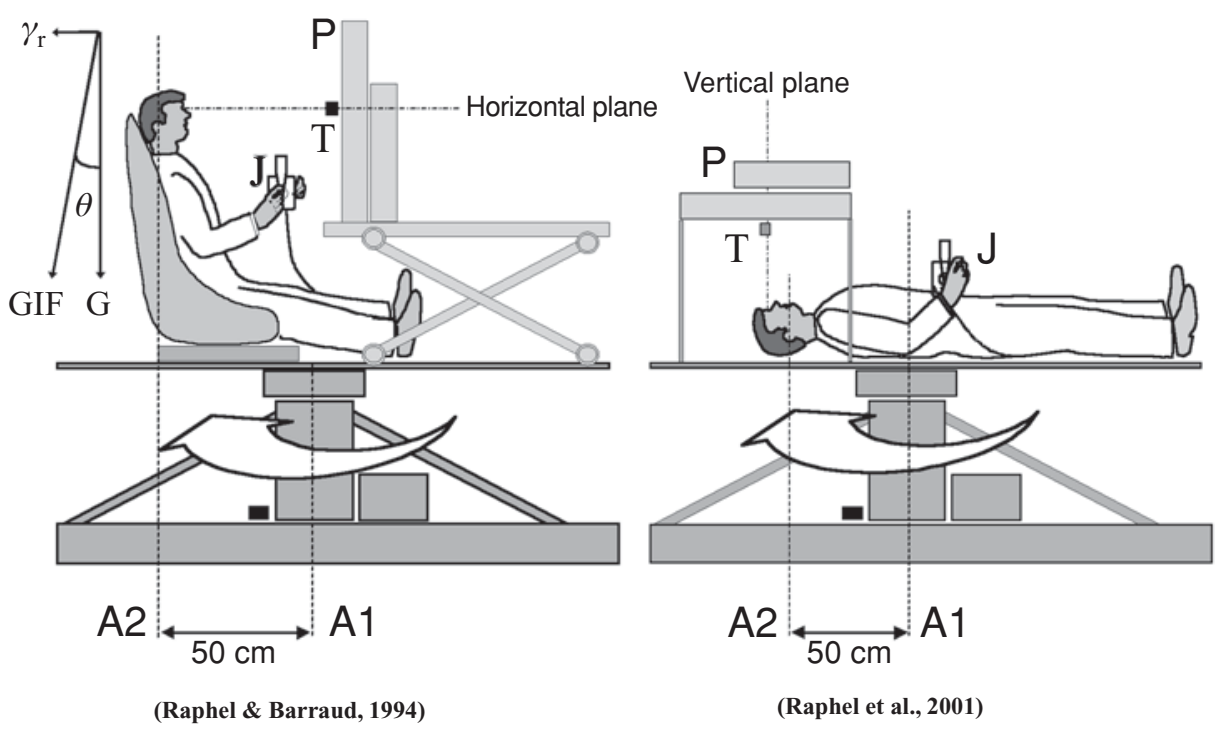

Figure 2. The apparatus consisted of a centrifuge in which the subject could take part in the experiment in the upright position (VPEL experiment; left panel) or in the supine position (VPAZ experiment; right panel). The illustration shows the distance between the axis of rotation (A1) and the axis of the subject's ear (A2), the electronic plotter board (P), the luminous target as a light-emitting diode (T), the joystick (J), and the horizontal and vertical planes passing through the eyes and the initial position of the LED. Also shown is the vectorial sum GIF between $G$ and radial acceleration $\gamma_{\mathrm{r}}$ under centrifugation. GIF is tilted at an angle with respect to the vertical. 
of the supine subjects, facing the zenith (Figure 2, right panel). For both experiments, the external auditory meatus was $50 \mathrm{~cm}$ from the axis of rotation. When the centrifuge rotated, the gravitational-inertial resultant force GIF applied on the subject was the vectorial sum of the radial horizontal acceleration $\gamma_{\mathrm{r}}$ combined with gravity $\mathrm{G}$. As a result, GIF was greater than $\mathrm{G}$ and tilted in pitch with respect to the vertical.

A luminous target, which was a yellow light-emitting diode (LED) fixed on the pencil holder of an electronic plotting board, was positioned $50 \mathrm{~cm}$ in front of the subject in a vertical position. Its accuracy of displacement was $\pm 0.05 \mathrm{~mm}$. The brightness of the LED $\left(2.82 \mathrm{~cd} / \mathrm{m}^{2}\right.$ measured by a SpectraScan 650$)$ was adjusted to be perceptible without dark accommodation. All the elements of the basic apparatus and the electronic plotting board were painted black and installed in a darkroom laboratory so that, when the LED was switched on, the subjects did not perceive any visual reference. With respect to their body reference, the subjects could move the LED both "above" and "below" and "to the right" and "to the left," using a joystick. LED motion on the electronic plotting board and centrifuge rotation velocity were computer controlled and monitored.

\section{Experimental Design}

Basically, we replicated the experiments of Raphel and Barraud (1994; VPEL) and Raphel et al. (2001; VPAZ). The VPEL experiment was conducted under five experimental conditions corresponding to five angular velocities: 0 (motionless), 10,20, 50, and $105^{\circ} / \mathrm{sec}$. The VPAZ experiment was conducted under six experimental conditions corresponding to the angular velocities of $0^{\circ}$ (motionless), $60^{\circ}, 80^{\circ}$, $100^{\circ}, 120^{\circ}$, and $140^{\circ} / \mathrm{sec}$. The data of radial accelerations, GIFs, and tilted angles of GIF with respect to G are summarized in Table 1 . Each condition was run in the same session in a growing order of angular velocity. Four target adjustments at eye level were made for each gravitational-inertial condition.

\section{Procedure}

Just before the session, subjects were instructed to use the joystick to set, in total darkness, the luminous target at the location perceived as the horizontal eye level in the VPEL experiment, or as the apparent zenith in the VPAZ experiment. Once the subjects were installed in the centrifuge, the middle of the plotter ( 0 on the $x y$ axis) was brought into alignment with the horizontal (VPEL experiment) or vertical (VPAZ experiment) median plane through the bridge of the nose (between the eyes). Subjects were further instructed to keep their head steady against the headrest at all times and a neck rest stabilized the position of the observer's head. Then, the laboratory lights were switched off, and 2 min afterward, subjects started by making four VPEL or VPAZ settings while remaining motionless and in total darkness. For each setting, the target was switched off and randomly displaced on both the $x$ and the $y$ axes in the periphery of the electronic plotting board. The LED was then switched on and a pip sound gave the signal to set the target to the VPEL or VPAZ and to validate the setting with a trigger on the joystick. Then, the LED was switched off again and displaced to the next preset position. Once the four settings in motionless were realized, the centrifuge motor was engaged and the centrifuge was started up slowly (acceleration $2 \% \mathrm{sec}^{2}$ ) until it rotated at $10^{\circ} / \mathrm{sec}$ (VPEL experiment) or $60^{\circ} / \mathrm{sec}$ (VPAZ experiment). After $2 \mathrm{~min}$ of rotation at constant velocity, subjects were asked to make the four settings (VPEL or VPAZ) under the given gravitational-inertial condition. Then, the rotating armchair was accelerated to the next velocity. At the end of the $105 \%$ sec condition (VPEL experiment) or $140^{\circ} / \mathrm{sec}$ condition (VPAZ experiment), the rotating armchair was stopped gradually.

\section{Data Collection}

All the measurements were made with respect to the center of the plotter ( 0 on the $x y$ axis). However, because the experimental effects expected on the VPEL or VPAZ concerned the $y$-axis, only the measurements made on this axis were taken into account in the data processing. For each gravitational-inertial condition, the VPEL or VPAZ was averaged over the four trials. The mean value measured while the subject was motionless and in total darkness served as a reference value, $\delta_{1}$. Experimental data in different centrifugation conditions consisted of the algebraic difference $\varepsilon$ between the VPEL or VPAZ measured under a gravitational-inertial condition $\left(\delta_{2}\right)$ and the reference value $\left(\delta_{1}\right): \varepsilon=\delta_{2}-\delta_{1}$. When $\varepsilon$ was negative, the VPEL or VPAZ was below the reference value, and when it was positive, the VPEL or VPAZ was above the reference value. The VPEL or VPAZ was expressed in degrees of visual angle. Data selected for statistical processing were $\varepsilon$. The relative measurement $\varepsilon$ was selected to remove any inaccuracy that might have been introduced as subjects were being installed on the apparatus - namely in terms of aligning with great precision the center of the plotter onto the gaze axis (i.e., nose bridge).

\section{RESULTS}

\section{Visually Perceived Eye Level Under Low Centrifugation}

In order to investigate the effects of radial accelerations and expertise on the VPEL, a 2 (groups: expert vs. control) $\times 4$ (radial accelerations, from 0.0152 to $1.67 \mathrm{~m} / \mathrm{sec}^{2}$ ) analysis of variance (ANOVA) with repeated measures on

Table 1

Experimental Conditions of Centrifugation With Respect to the Different Saccular and Utricular Thresholds and Physic Resultant Values Calculated for Each Gravito-Inertial Condition

\begin{tabular}{ccccc}
\hline Experiment & $\begin{array}{c}\text { Rotation } \\
\text { Velocity } \\
(\mathrm{deg} / \mathrm{sec})\end{array}$ & $\begin{array}{c}\text { Radial } \\
\text { Acceleration } \gamma_{\mathrm{r}} \\
\left(\mathrm{m} / \mathrm{sec}^{2}\right)\end{array}$ & $\begin{array}{c}\text { Gravito-Inertial } \\
\text { Force GIF } \\
\left(\mathrm{m} / \mathrm{sec}^{2}\right)\end{array}$ & $\begin{array}{c}\text { Tilted Angle } \theta \text { of } \\
\text { GIF With Respect } \\
\text { to G }(\mathrm{deg})\end{array}$ \\
\hline VPEL & $\begin{array}{c}\text { Motionless } \\
10\end{array}$ & 0 & 9.810 & 0 \\
& 20 & 0.015 & 9.810012 & 0.09 \\
VPAZ & 50 & 0.061 & 9.810189 & 0.36 \\
& 105 & 1.67 & 9.817372 & 2.22 \\
& Motionless & 0 & 9.951 & 9.66 \\
& 60 & 0.55 & 9.810 & 0 \\
& 80 & 0.97 & 9.825 & 3.21 \\
& 100 & 1.52 & 9.858 & 5.64 \\
& 120 & 2.19 & 9.927 & 8.81 \\
& 140 & 2.98 & 10.050 & 12.60 \\
\hline
\end{tabular}


the last factor was applied to the mean visual deviation from the reference value $(\varepsilon)$. Results showed main effects of expertise $[F(1,22)=23.3 ; p<.001]$ and GIF conditions $[F(3,66)=219.47 ; p<.001]$ and an interaction of the two factors $[F(3,66)=18.74 ; p<.001]$. A post hoc analysis (Newman-Keuls test) showed that increasing the radial acceleration lowered the VPEL - that is a shift downward toward the lower part of the body as indicated by the negative values (Table 2)-more consistently for the control subjects than for the experts $(p<.05)$. Moreover, a reference (VPEL motionless) comparison $t$ test showed that the VPEL measures became lower than the reference value with the $0.0609 \mathrm{~m} / \mathrm{sec}^{2}$ radial acceleration $(t=-2.77, p<.01)$ for the control subjects. For the expert subjects, the VPEL measures were lower than the reference only for the $1.67 \mathrm{~m} / \mathrm{sec}^{2}$ radial acceleration $(t=$ $-6.34, p<.001)$.

In addition, significant relationships were found between the radial acceleration $\gamma_{\mathrm{r}}$ and the lowering of the VPEL for both groups (Figure 3, top panel). This relationship can be described by the logarithmic regression equations: $d \gamma_{\mathrm{r}}=-1.63 \ln \gamma_{\mathrm{r}}-6.59\left(R^{2}=.81\right)$ for the control group and $d \gamma_{\mathrm{r}}=-0.67 \ln \gamma_{\mathrm{r}}-2.48\left(R^{2}=.70\right)$ for the expert group, where $d \gamma_{\mathrm{r}}$ (in degrees) is the decrease in the visual angle of the VPEL, relative to the VPEL reference, and $\gamma_{\mathrm{r}}$ (in meters per second squared) is the radial acceleration of centrifugation. The expert group showed a higher intercept and a weaker slope. Thus, the theoretical threshold of sensitivity to radial acceleration $(y=0)$ was higher for the experts than for the controls (0.02 vs. $\left.0.052 \mathrm{~m} / \mathrm{sec}^{2}\right)$ whereas the VPEL variations were higher (and therefore, more noticeable) for the control than for the expert group.

\section{Visually Perceived Apparent Zenith Under Low Centrifugation}

In order to investigate the effects of radial accelerations and expertise on the VPAZ, a 2 (groups: expert vs. control) $\times 5$ (radial accelerations, from 0.55 to $2.98 \mathrm{~m} /$ $\mathrm{sec}^{2}$ ) ANOVA with repeated measures on the last factor was applied to the mean visual deviation from the reference value $(\varepsilon)$. Results showed main effects of expertise $[F(1,14)=6.37, p<.05)]$, no effect of centrifugation conditions $[F(4,56)=2.29, p>.05]$ but an interaction of the two factors $[F(4,56)=3.33, p<.05]$. As illustrated in Table 3, increasing the radial acceleration lowered the VPAZ for the control subjects $(p<.001)$, whereas there was no difference for the VPAZ judgments according to the radial accelerations for the experts $(p<.6)$. For the control subjects, the VPAZ perceived under each centrifugation condition was lower than the reference motionless ( $t$ tests; $t>4.64, p<.01$ ), that is, shifted upward toward the lower part of the body as indicated by the negative values. Whereas, for the expert subjects, the VPAZ perceived under each centrifugation condition was not different from the VPAZ reference $(t<0.71, p>.10)$. Thus, the expert group was not sensitive to low gravitationalinertial stimulation.

A significant relationship was found between the radial acceleration $\gamma_{\mathrm{r}}$ and the lowering of VPAZ for the control group $\left(R^{2}=.75\right)$ but not for the expert group $\left(R^{2}=.006\right)$. This relationship (Figure 3, bottom panel) was described by the logarithmic regression equation: $d \gamma_{\mathrm{r}}=-2.35 \ln \gamma_{\mathrm{r}}-5.2$, where $d \gamma_{\mathrm{r}}$ (in degrees) is the decrease in the visual angle of the VPAZ, relative to the VPAZ reference, and $\gamma_{\mathrm{r}}$ (in meters per seconds squared) is the radial acceleration of centrifugation. The theoretical threshold of sensitivity to radial acceleration for the control group was $0.11 \mathrm{~m} / \mathrm{sec}^{2}$.

\section{DISCUSSION}

The aim of the present study was to investigate the influence of low gravitational-inertial stimulation on the perception of target elevation through the level of subject's spatial expertise.

\section{Effect of Expertise on the Perception of Target Elevation}

According to the literature, the "control" observers set the target (VPEL and VPAZ) progressively lower as the magnitude of GIF increased. Thus, a target that remained at true level would appear to be more elevated with each increase in GIF. Moreover, the perception of the eye level corresponded to a logarithmic psychophysical function, but the theoretical thresholds of sensitivity to radial accelerations were different from those already described in the literature for similar experimental conditions. The theoretical threshold - that is, the radial acceleration of centrifugation observed when the deviation of the subjective reference was null (i.e., $\gamma_{\mathrm{r}}$ for $d \gamma_{\mathrm{r}}=0$ ) - was higher in the upright position $\left(0.02 \mathrm{~m} / \mathrm{sec}^{2}\right.$ vs. $0.0006 \mathrm{~m} / \mathrm{sec}^{2}$; Raphel \& Barraud, 1994) whereas it was lower in the supine position $\left(0.11 \mathrm{~m} / \mathrm{sec}^{2}\right.$ vs. $0.38 \mathrm{~m} / \mathrm{sec}^{2}$; Raphel et al., 2001). These differences could be connected to the interindividual variability, which is the main purpose of the present work. Indeed, the gravitational-inertial disturbances did not induce the same negative consequences

Table 2

Mean Deviation (in Degrees) and Standard Deviation of VPEL Settings Relative to the VPEL Reference (Motionless) for the Four Conditions of Radial Acceleration

\begin{tabular}{|c|c|c|c|c|c|c|c|c|}
\hline \multirow[b]{3}{*}{ Group } & \multicolumn{8}{|c|}{ Conditions of Radial Acceleration } \\
\hline & \multicolumn{2}{|c|}{$0.02 \mathrm{~m} / \mathrm{sec}^{2}$} & \multicolumn{2}{|c|}{$0.06 \mathrm{~m} / \mathrm{sec}^{2}$} & \multicolumn{2}{|c|}{$0.38 \mathrm{~m} / \mathrm{sec}^{2}$} & \multicolumn{2}{|c|}{$1.67 \mathrm{~m} / \mathrm{sec}^{2}$} \\
\hline & $M$ & $S D$ & $M$ & $S D$ & $M$ & $S D$ & $M$ & $S D$ \\
\hline Control & -0.79 & 1.64 & -1.40 & 1.75 & -3.07 & 1.56 & -8.95 & 1.89 \\
\hline Expert & 0.28 & 0.89 & 0.30 & 1.01 & -0.26 & 1.16 & -4.02 & 2.20 \\
\hline
\end{tabular}




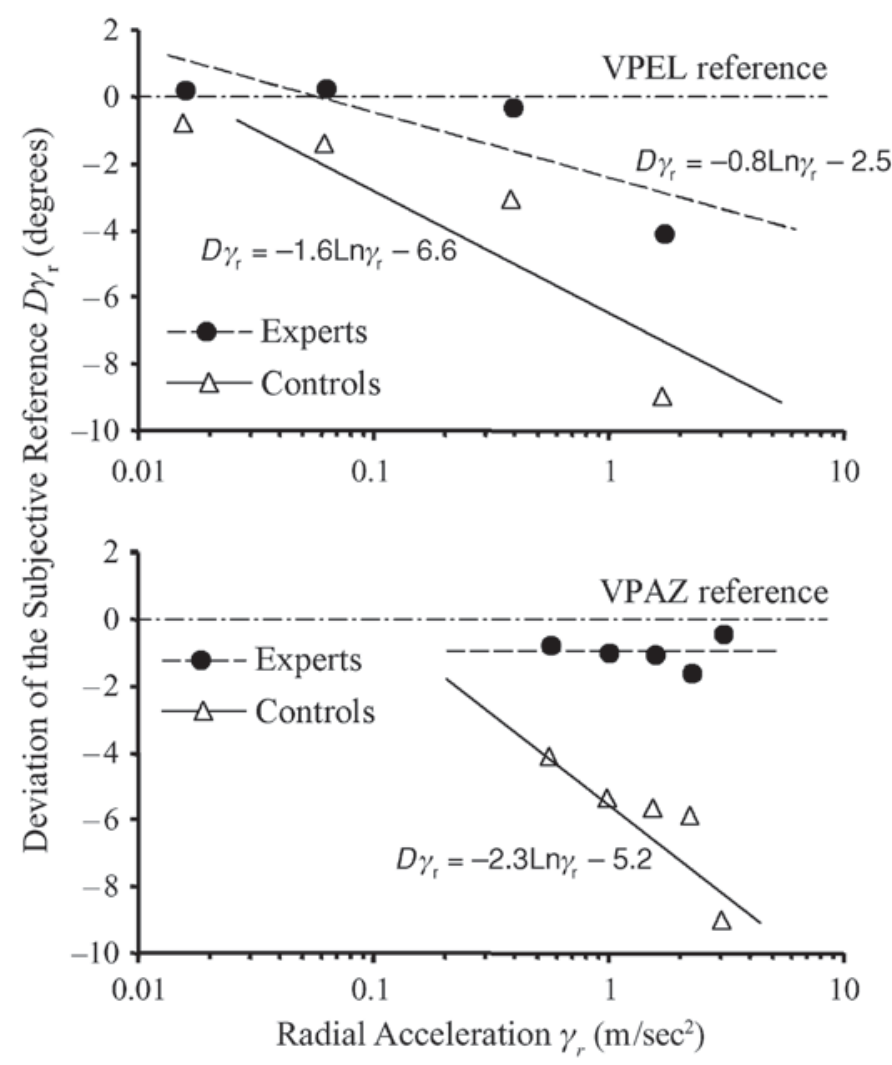

Figure 3. Lowering of the VPEL (top panel) and VPAZ (bottom panel) for the expert and control groups (logarithmic scale). $D \gamma_{\mathrm{r}}$ is the logarithmic function of radial acceleration magnitude $\left(\gamma_{r}\right)$ under low centrifugation. Zero on the $\mathrm{Y}$ axis is the visually perceived reference value for curves while the subject is motionless and in darkness.

on the eye level for the spatial experts. In the supine position, acrobats were not subjected to illusion for the radial accelerations up to $2.98 \mathrm{~m} / \mathrm{sec}^{2}$ ( $\left.\mathrm{GIF}=10.25 \mathrm{~m} / \mathrm{sec}^{2}\right)$. There was a significant VPEL lowering in comparison with motionless for the radial acceleration of $1.67 \mathrm{~m} / \mathrm{sec}^{2}$ $\left(\mathrm{GIF}=9.95 \mathrm{~m} / \mathrm{sec}^{2}\right)$; however, this apparent target elevation was much smaller than for the control subjects. In the upright position, the spatial experts showed a smaller sensitivity to the oculogravic illusion observed at the level of the theoretical threshold of radial accelerations sensitivity $\left(0.052 \mathrm{~m} / \mathrm{sec}^{2}\right)$ but also at the level of the oculogravic illusion scale.

\section{Functional Enhancement of the Vestibular System With Respect to Subjects' Spatial Experience}

The VPEL and VPAZ correspond, respectively, to the horizontal and vertical planes subjectively defined by the individual as being perpendicular or parallel to the gravity and passing by the center of the eye lenses. During low GIF stimulation, under the somaesthetic stimulation threshold, the perceptive shift could be due to the otolithic incapacity to distinguish linear acceleration from head-body tilt. The control subjects may have interpreted the otolithic stimulation as a back tilt. Conversely, it is possible that the absence of oculogravic illusion for the spatial experts could result from a functional enhancement of the otolithic system, which can resolve the stimulus ambiguities between linear acceleration and head-body tilt. Indeed, significant differences in ocular counterrolling between roll-tilt and centrifugation in the upright position have been observed (MacDougall, Curthoys, Betts, Burgess, \& Halmagyi, 1999). This result suggests that, at a constant interaural shear force, the dorsoventral shear that differed between tilt and translation (probably predominantly the saccular stimulation; Curthoys et al., 1999) assists in the dissociation of tilts from translations. Thus, the combined effect of interaural and dorsoventral stimulation was taken into account to resolve the apparent otolithic stimulation ambiguities. However, there were large individual differences when comparing the ocular torsion in the centrifugation and tilt-chair conditions (MacDougall et al., 1999) suggesting that, for some subjects, linear acceleration and head tilt are not distinguishable.

Within that theoretical framework, it can be suggested that the spatial experts were less sensitive to the oculogravic illusion because of their otolithic capacity to distinguish linear accelerations from a head-body tilt. However, a more general explanation simply suggests that different thresholds and sensitivities to the inertial changes may 
Table 3

Mean Deviation (in Degrees) and Standard Deviation of VPAZ Settings Relative to the VPAZ Reference (Motionless) for the Five Conditions of Radial Acceleration

\begin{tabular}{|c|c|c|c|c|c|c|c|c|c|c|}
\hline \multirow[b]{3}{*}{ Group } & \multicolumn{10}{|c|}{ Conditions of Radial Acceleration } \\
\hline & \multicolumn{2}{|c|}{$0.55 \mathrm{~m} / \mathrm{sec}^{2}$} & \multicolumn{2}{|c|}{$0.97 \mathrm{~m} / \mathrm{sec}^{2}$} & \multicolumn{2}{|c|}{$1.52 \mathrm{~m} / \mathrm{sec}^{2}$} & \multicolumn{2}{|c|}{$2.19 \mathrm{~m} / \mathrm{sec}^{2}$} & \multicolumn{2}{|c|}{$2.98 \mathrm{~m} / \mathrm{sec}^{2}$} \\
\hline & $M$ & $S D$ & $M$ & $S D$ & $M$ & $S D$ & M & $S D$ & $M$ & $S D$ \\
\hline & -4.10 & 1.83 & -536 & 3.12 & -564 & 2.46 & -5.88 & & -9.01 & 4.23 \\
\hline Expert & -0.71 & 4.31 & -0.95 & 4.67 & -1.00 & 5.49 & -1.56 & 6.16 & -0.40 & 6.57 \\
\hline
\end{tabular}

themselves, at least to some extent, account for the difference between acrobats and controls on the visual illusion. In other words, experts may have habituated in general to acceleration, and in any task that requires a judgment that could be influenced by acceleration, they would exhibit a lower sensitivity to acceleration.

A study done by Merfeld, Zupan, and Peterka (1999) put in evidence that predictive internal models are at the origin of the perceptive distinction between gravity and other linear accelerations. The sensitivity with which subjects perceive a variation of sensory state would depend on the comparison of the signals related to body orientation with predictive internal models specifying expected sensory configurations (Berthoz \& Viaud-Delmon, 1999). From a body perception and control orientation point of view, these internal representations were elaborated from the subjects' experience (Lackner, 1992; Lestienne \& Gurfinkel, 1988; Young, Mendoza, Groleau, \& Wojcik, 1996). Their adequacy to an environmental reality would be, then, different from one individual to another. In that way, extensive acrobatic practice requires the ability to associate unusual sensory configurations with a precise body orientation. Through learning, new configurations corresponding to postural states would be stored at the level of the central nervous system, enriching the internal models of orientation. Within that context, the enhancement of the experts' spatial internal models could abolish the sensory conflict that leads to a perceptive confusion between linear accelerations and body tilt.

\section{Sensory Weighting With Respect to Subjects' Spatial Experience}

However, this interpretation in terms of otolithic system enhancement for the acrobats could not completely explain the presence of the oculogravic illusion for the stronger accelerations (in upright position). Another hypothesis can be advanced. The object location above or below the eye level requires information about the body orientation of the subject. In the absence of vision, the perception of body orientation in the gravitational field would be based on the coordination of vestibular and somaesthetic sensory modalities. In the classical conception, vestibular information was long considered an essential sensory support and the source of dominant information for "the static orientation with regard to the vertical" (Young, 1984). However, the somaesthetic system is assumed to provide information about body orientation, notably in response to the antigravitational forces. Most of the time, the information provided by these two systems (vestibular and somaesthetic) is redundant. Then, the errors of perceptive judgment would depend on an inadequate resolution of a sensory conflict generated by the presence of clashing or nonredundant information, implying an inappropriate sensory dominance during the integration process (Young, 1984). Within this theoretical context, at constant velocity, the eye level lowering observed for the very low gravitational-inertial stimulation may be the result of a sensory conflict between the otolithic information, for which the sensors do not distinguish a linear acceleration from a head tilt, and the somaesthetic information, which indicates no change in the postural state with regard to gravity. The perceptive shift then would be associated with a process of sensory integration, which gives more weight to the otolithic information and interprets the gravitational modifications in terms of physical tilt. Conversely, the absence of oculogravic illusion may be the result of a somaesthetic sensory dominance. The literature underlines that the acquired experience by the acrobats could increase the somaesthetic sensitivity of these subjects and more particularly the pregnancy of the gravity somaesthetic cues (Bringoux et al., 2000; Lackner, 1992; Lestienne \& Gurfinkel, 1998; Vuillerme et al., 2001).

This assumption of individual sensorial weighting could explain why there appeared to be no difference between pilots (considered as experts) and nonpilots in the reported strength of their oculogravic illusions (Cohen, Crosbie, \& Blackburn, 1973). Indeed, in this study, important GIF variations were used (4 G during 2-3 sec). Under such conditions, the vestibular system was stimulated, but cutaneous and other mechanoreceptors were also strongly stimulated by the deformation engendered by the acceleration (Benson, 1990). In the present study, low GIF variations were used (1 to $1.05 \mathrm{G}$ ) so that only the vestibular system was stimulated. Thus, it was possible to estimate the importance that each individual attributes to this input. Nevertheless, the radial acceleration of $1.67 \mathrm{~m} /$ $\sec ^{2}$ led a VPEL shift, although smaller than for the control subjects. For this GIF and for a subject of $70 \mathrm{~kg}$, the variation of subject's weight was $1 \mathrm{~kg}$ approximately. This variation has been generally considered as a negligible quantity. However, it is possible that with experience, the acrobats are able to detect these subtle cues and use them for the perception of body orientation. In other words, the VPEL shift would have a tactile and kinesthetic origin for spatial experts.

In conclusion, in the absence of environmental visual cues, vestibular stimulation is responsible for the per- 
ceptive construction that leads to the unconscious visual target elevation. However, the relations that the subject maintains with the spatial environment and the knowledge acquired through experience modify the processing of sensory information and the perceptive construction resulting from it. The extensive practice of acrobatics, which requires one to finely associate sensory configurations with a precise physical orientation, allows these spatial experts to be less sensitive to the oculogravic illusion. It is difficult, however, to determine whether these interindividual differences have as their origin a sensory dominance or a better efficiency in the use of the available sensory information. Future research should investigate the effects of higher gravitational-inertial stimulation applied to the vestibular sensors as well as to the mechanoreceptive proprioception. It should also investigate to what extent acrobat experts exhibit lower sensitivity thresholds for noticing that an acceleration is taking place.

\section{REFERENCES}

Benson, A. J. (1990). Sensory functions and limitations of the vestibular system. In R. Warren \& A. H. Wertheim (Eds.), Perception and control of self-motion (pp. 145-170). Hillsdale, NJ: Erlbaum.

Berthoz, A. (1997). Le sens du mouvement. Paris: Odile Jacob.

Berthoz, A., \& Viaud-Delmon, I. (1999). Multisensory integration in spatial orientation. Current Opinion in Neurobiology, 9, 708-712.

Bringoux, L., Marin, L., Nougier, V., Barraud, P. A., \& Raphel, C. (2000). Effects of gymnastics expertise on the perception of body orientation in the pitch dimension. Journal of Vestibular Research, 10, 251-258

Citek, K., \& Ebenholtz, S. M. (1996). Vertical and horizontal eye displacement during static pitch and roll postures. Journal of Vestibular Research, 6, 213-228.

COHEN, M. (1973). Elevator illusion: Influences of otolith organ activity and neck proprioception. Perception \& Psychophysics, 14, 401-406.

Cohen, M. (1981). Visual-proprioceptive interactions. In R. D. Walk \& H. L. Pick (Eds.), Intersensory perception and sensory integration (pp. 175-216). New York: Plenum.

Cohen, M., Crosbie, R. J., \& Blackburn, L. H., (1973). Disorienting effects of aircraft catapult launchings. Aviation, Space, \& Environmental Medicine, 44, 37-39.

Cohen, M., Stoper, A., Welch, R., \& DeRoshia, C. (2001). Effects of gravitational and optical stimulation on the perception of target elevation. Perception \& Psychophysics, 63, 29-35.

Correia, M. J., Hixon, W. C., \& Niven, J. I. (1968). On predictive equations for subjective judgments of vertical and horizontal in a force field. Acta Oto-Laryngologica (Suppl. 230).

Curthoys, I. S., Betts, G. A., Burgess, A. M., MacDougall, H. G., Cartwright, A. D., \& Halmagyi, G. M. (1999). The plane of the utricular and saccular maculae of the guinea pig. Annals of the New York Academy of Sciences, 871, 27-34.

Dizio, P., Li, W., Lackner, J. R., \& Matin, L. (1997). Combined influences of gravito-inertial force level and visual field pitch on visually perceived eye level. Journal of Vestibular Research, 7, 381-392.

Graybiel, A. (1952). Oculogravic illusion. Archives of Ophthalmology, 8, 605-615.

Kelly, J. P. (1991). The sense of balance. In E. R. Kandel, J. H. Schwartz, \& T. M. Jessell (Eds.), Principles of neural science (p. 503). New York: Elsevier.

LACKNER, J. R. (1992). Multimodal and motor influences on orientation: Implications for adapting to weightless and virtual environments. Journal of Vestibular Research, 2, 307-322.

Lestienne, F., \& GuRfinKel, V. S. (1988). Postural control in weightlessness: A dual process underlying adaptation to an unusual environment. Trends in Neurosciences, 11, 359-363.
LI, W., \& Matin, L. (1993). Eye and head position, visual pitch, and perceived eye level. Investigative Ophthalmology \& Visual Science, 34, 311 .

Li, W., \& Matin, L. (1995). Differences in influence between pitchedfrom-vertical lines and slanted-from-frontal horizontal lines on egocentric localization. Perception \& Psychophysics, 57, 71-83.

MacDougall, H. G., Curthoys, I. S., BetTs, G. A., Burgess, A. M., \& HalmagYi, G. M. (1999). Human ocular counterrolling during roll-tilt and centrifugation. Annals of the New York Academy of Sciences, 871, 173-180.

Matin, L. (1982). Visual localization and eye movements. In A. Wertheim, W. A. Wagenaar, \& H. Leubowitz (Eds.), Tutorials on motion perception. New York: Plenum.

Matin, L. (1986). Visual localization and eye movements. In K. R. Boff, L. Kaufman, \& J. P. Thomas (Eds.), Handbook of perception \& human performance (Vol. 1, pp. 20-1 to 20-45). New York: Wiley.

Matin, L., \& Fox, C. R. (1989). Visually perceived eye level and perceived elevation of objects: Linearly additive influences from visual field pitch and from gravity. Vision Research, 29, 315-324.

Matin, L., \& LI, W. (1992). Visually perceived eye level: Changes induced by pitched-from-vertical 2-line visual field. Journal of Experimental Psychology: Human Perception \& Performance, 18, 257-289.

Matin, L., \& LI, W. (1994). The influence of a stationary single line in darkness on the visual perception of eye level. Vision Research, 34, 311-330.

Matin, L., \& LI, W. (1995). Multimodal basis for egocentric spatial localization and orientation. Journal of Vestibular Research, 5, 499-518.

Merfeld, D. M., Zupan, L., \& Peterka, R. J. (1999). Humans use internal models to estimate gravity and linear acceleration. Nature, 398, 615-618.

Miller, E. F., \& Graybiel, A. (1966). Magnitude of gravitational-inertial force: An independent variable in egocentric visual localization of horizontal. Journal of Experimental Psychology, 71, 452-460.

Raphel, C., \& Barraud, P. A. (1994). Perceptual thresholds of radial acceleration as indicated by visually perceived eye level. Aviation, Space, \& Environmental Medicine, 65, 204-208.

Raphel, C., Barraud, P. A., Koessler, C., \& Cian, C. (1996). Effects of frame and low radial accelerations to the visually perceived eye level. Perception \& Psychophysics, 58, 906-914.

Raphel, C., Cian, C., Barraud, P. A., \& Micheyl, C. (2001). Effects of supine body position and low radial accelerations on the visually perceived apparent zenith. Perception \& Psychophysics, 1, 36-46.

SCHÖNE, H. (1964). On the role of gravity in human spatial orientation. Aerospace Medicine, 35, 764-772.

Stoper, A. E., \& Cohen, M. M. (1989). Effects of structured visual environments on apparent eye level. Perception \& Psychophysics, 46, 469-475.

Stoper, A. E., \& Cohen, M. M. (1991). Optical, gravitational and kinaesthetic determinants in judged eye level. In S. R. Ellis (Ed.), Pictorial communication in virtual and real environments (pp. 391-403). London: Taylor \& Francis.

Vuillerme, N., Danion, F., Marin, L., Boyadjian, A., Prieur, J. M., Weise, I., \& Nougier, V. (2001). The effect of expertise in gymnastics on postural control. Neuroscience Letters, 303, 83-86.

WADE, N. J., \& SchÖNE, H. (1971). The influence of force magnitude on the perception of body position: I. Effects of head posture. British Journal of Psychology, 62, 157-163.

Whiteside, T. C. D., Graybiel, A., \& Niven, J. I. (1965). Visual illusions of movement. Brain, 88, 193-210.

YounG, L. R. (1984). Perception of the body in space: Mechanisms. In I. Darian-Smith (Vol. Ed.), Handbook of physiology: Section 1. The nervous system: Vol. 3. Sensory processes (pp. 1023-1066). New York: Academic Press.

Young, L. R., Mendoza, J. C., Groleau, N., \& Wojcik, P. W. (1996). Tactile influences on astronaut visual spatial orientation: Human neurovestibular studies on SLS-2. Journal of Applied Physiology, 81, $44-49$

(Manuscript submitted June 13, 2003; revision accepted for publication October 18, 2004.) 